\title{
Development of mobile robots based on peristaltic crawling of an earthworm
}

\author{
Hayato Omori, Taro Nakamura, \\ Tomohide Iwanaga and Takeshi Hayakawa \\ Chuo University, Department of Precision Mechanics
}

Japan

\section{Introduction}

An earthworm moves by peristaltic crawling. It produces extension and contraction waves along the anteroposterior direction, from the anterior to posterior, by varying the thickness and length of its segments. This locomotion mechanism is in contact with a large area on the ground when the robot moves. Furthermore, it requires less space than other locomotion mechanisms, e.g. bipedal, wheel-based or meandering snake-like locomotion. Therefore, this mechanism is attractive not only for rescue and exploration robots operating in constrained environments but also for locomotive endoscope robots in the field of medical engineering. In recent years, some robotic and biomedical engineering researchers have developed peristaltic crawling robots based on earthworm locomotion. A prototype microrobot was developed, which moved using an external magnetic field (Saga et al., 2004). This robot consisted of individual cells made of natural latex rubber tubes with a water-based magnetic fluid sealed inside, corresponding to an earthworm's segments. This peristaltic crawling robot was developed for rescue applications (Saga et al., 2004). The robot is actuated by an artificial rubber muscle that is driven by a pneumatic system (Nakamura et al., 2003). Because this artificial muscle is reinforced longitudinally by Kevlar fibres, the actuator not only contracts in the longitudinal direction, but when internal pressure is applied, it also expands in the radial direction. This robot achieves peristaltic crawling by employing this muscle for segment contraction and expansion. Zou et al. (2005) proposed an earthworm-like microrobot for intestinal inspection. This microrobot consists of several extensor units that are actuated by a micro DC motor and a screw-driven device. Actuating the extensor units in an appropriate sequence allows the microrobot to propel itself. The above robot studies apply various actuation methods to achieve locomotion by peristaltic crawling. However, the motion velocity of these robots is very low and inefficient. Furthermore, only movement through a straight pipe has undergone experimental testing. The influences of various conditions on peristaltic crawling locomotion patterns of earthworm robots must be examined systematically to allow stable adjustment to environmental variations.

In this study, we developed two types of prototype robots based on peristaltic crawling of an earthworm and then introduce one of these as an earthworm robot. One is called the 'flexible-type robot', and consists of flexible units; the other is called the 'rigid-type robot', 
which is able to bend between units. We proposed the flexible-type robot for moving passively in narrow spaces such as curved pipes, and the rigid-type robot for moving along given routes on a plane surface. In this paper, we first explain peristaltic crawling of an earthworm, which was analysed for comparison with the locomotion of the proposed robots. Using the flexible-type robot, we performed experiments with forward and turning movements on a plane surface, as well as in a curved pipe, to confirm whether the flexible units suit both environments. We propose two locomotion patterns for turns. The rigid-type robot was modelled to evaluate its position and attitude, and a simulation analysed its movement for given routes on a plane surface. We paid particular attention to the actual movement of an earthworm in dirt and developed an underground explorer robot. This unit was made of parallel links, and we performed experiments in dirt, which showed good performance.

\section{Peristaltic crawling motion of an earthworm}

\subsection{The structure of an earthworm and its peristaltic motion mechanism}

The inner structure of an earthworm is shown in Fig. 1. The earthworm consists of numerous segments divided by septa and a coelom containing the alimentary canal and nerve circuits. The inner wall of the body is composed of two muscle layers. The outside layer is called the circular muscle and the inside one is called the longitudinal muscle. When the circular muscle is actuated in a radial direction, the segment becomes thinner and extends in the axial direction. When the longitudinal muscle contracts in the axial direction, the segment gets thicker and shorter.

Fig. 2 shows the locomotion pattern of an earthworm during peristaltic crawling. The earthworm propagates a longitudinal wave from the anterior to posterior part using contraction muscles in consecutive segments. The locomotion pattern can be described as follows:

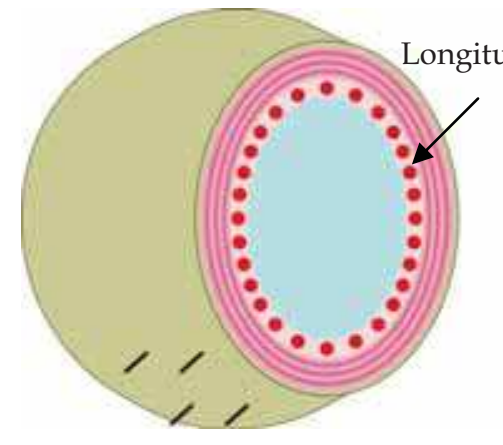

a) Contraction

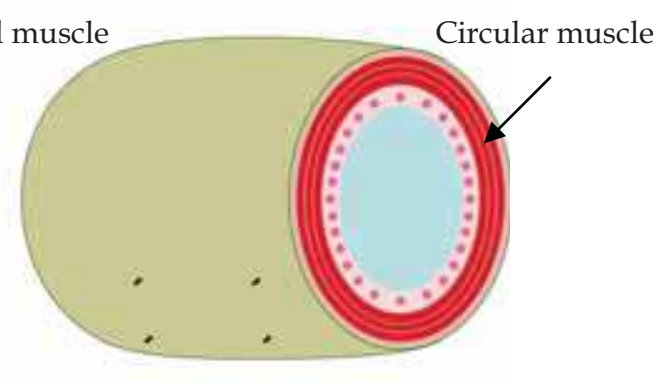

a) Extension

Fig. 1. Inner structure of an earthworm

1) The anterior segments of the earthworm are contracted by the longitudinal muscle. In this case, because the thicker segments are in contact with the surface during locomotion, 
the friction between the segments and the ground is increased. Therefore, the segments remain in contact with the surface.

2) The contraction continuously propagates to the rear end. This movement pulls the rear segments in the direction of movement.

3) The anterior segments of the earthworm are extended in the axial direction by the circular muscle. In this case, since the friction between the segments and the moving surface is reduced, thinner segments can move smoothly. Furthermore, because the rear segments remain in contact with the surface, the thinner segments can move forward.

4) The extended segments continuously propagate in the rear direction. The forward segments are pushed in the direction of movement.
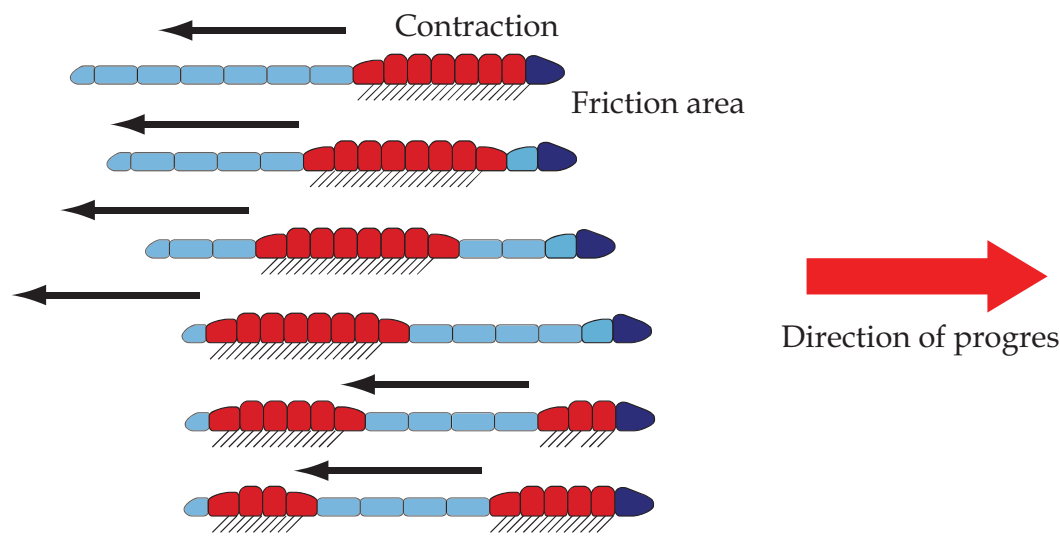

Direction of progress

Fig. 2. Locomotion pattern of an earthworm

\subsection{Measurement of an actual earthworm's peristaltic crawling}

The motion of an actual earthworm was compared with the peristaltic crawling motion of the locomotion robot. A 140-mm-long and 4-mm-thick earthworm was used. Marks were placed on the earthworm's segments every $19 \mathrm{~mm}$ (Fig. 3). The locomotion was videotaped with a digital video camera and analysed using a movement analysis software (MOVIAS Pro: Nac Image Technology Inc.). The results obtained are shown in Fig. 4.

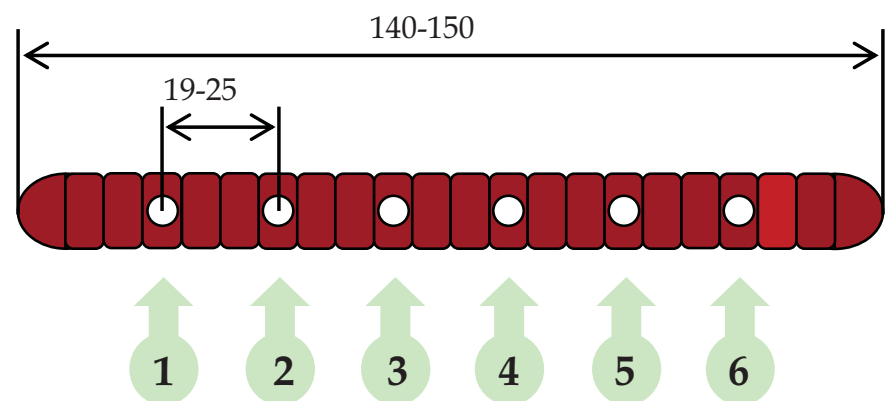

Fig. 3. Earthworm segments and markers 
This figure shows that contraction begins from the anterior part of the earth-worm and continuously propagates towards the posterior part. The anterior segments contract again, after propagation to the posterior part is complete. The average velocity of the earthworm was $16.1 \mathrm{~mm} / \mathrm{s}$. The peristaltic crawling robot was designed by taking these results into consideration.

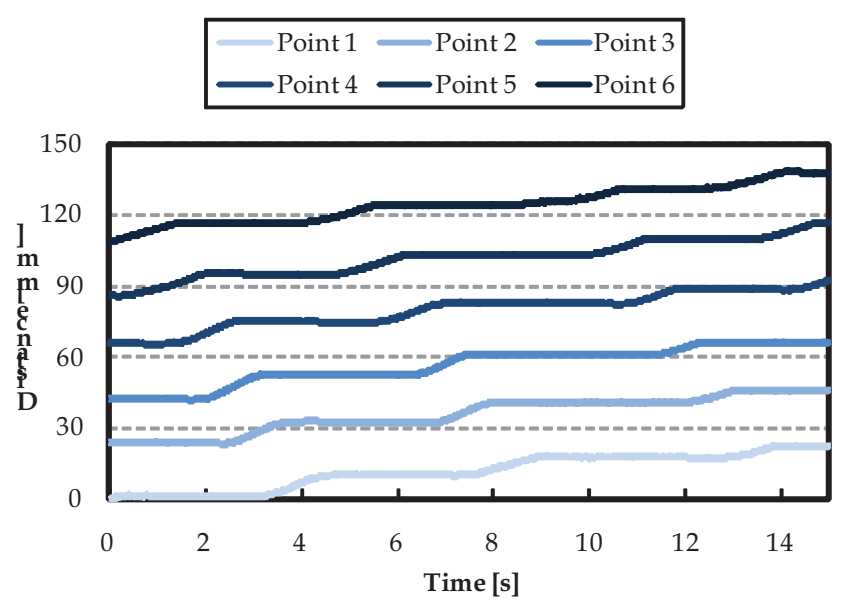

Fig. 4. Peristaltic crawling motion of the actual earthworm

\section{Flexible-type robot}

\subsection{Configuration of a Robot with 7 Units}

The peristaltic crawling robot of flexible type was developed by referring to an actual earthworm's motion. Fig. 5 shows the configuration of the robot. The complex mechanism of an actual earthworm was simplified. A unit of the robot is equal to a segment of an actual earthworm. Since peristaltic crawling requires at least 3 units, it has 7 units. Each of them can extend and contract in the axial direction, which is necessary to realise peristaltic crawling. A unit is actuated by servomotors controlled by the pulse signal of PIC. Rubber sheets are attached to each belt for generating friction between the robot and the ground or inner wall of a tube.

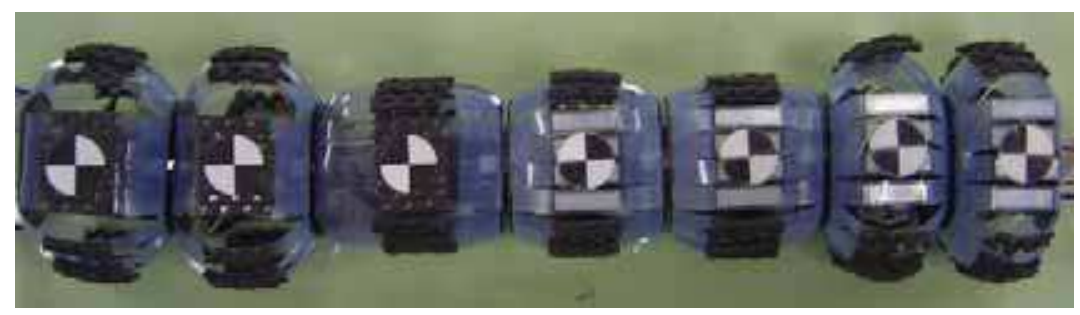

Fig. 5. Flexible-type robot 
The length of 7 units is $386 \mathrm{~mm}$ when fully extended and $295 \mathrm{~mm}$ when fully contracted. The robot weights $341 \mathrm{~g}$. Marks are placed on each unit, which are captured in a video, and then the movements of the robot are analysed.

\subsection{The Structure of a Flexible Unit}

The structure of a unit is shown in Fig. 6. It contains 2 servomotors, whose rotation is transmitted to the cranks and plate A is shifted back and forth. Therefore, the unit expands in the radial direction during contraction due to the curvature of the belts made of flexible plates (Fig. 7).

One of the features of the unit is flexibility. Plate A can be tilted by changing the angle of the servomotor (Fig. 7). A contraction, an extension and a tilt plate are smoothly realised by adopting a leaf spring as a crank. The tilt angle ranges from -20 to 20 degrees. Therefore, the robot has flexibility for environment shape (Fig. 8).
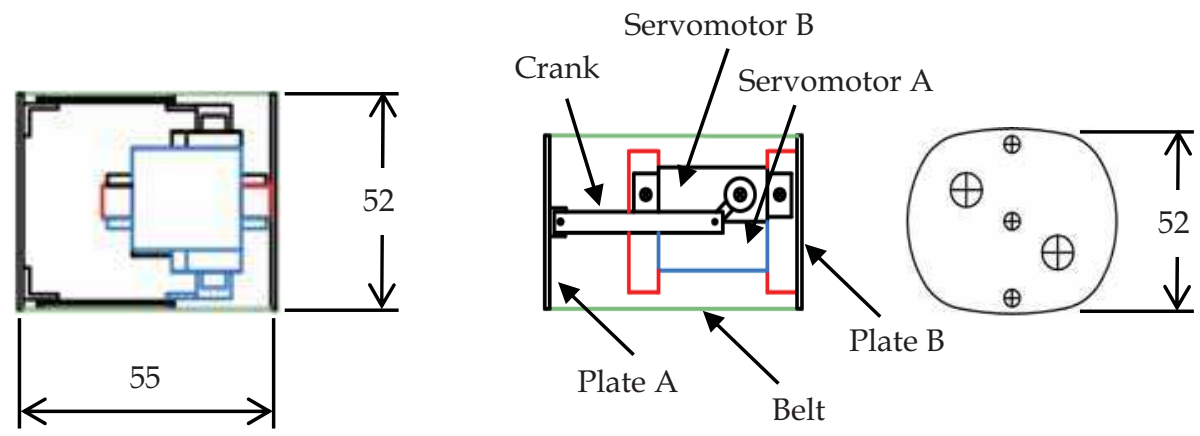

Fig. 6. Structure of a unit

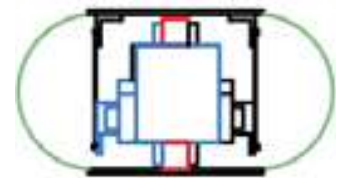

Contraction

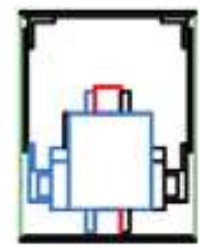

Extension

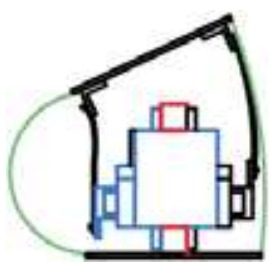

Angle control

Fig. 7. Contraction, extension and angle control 


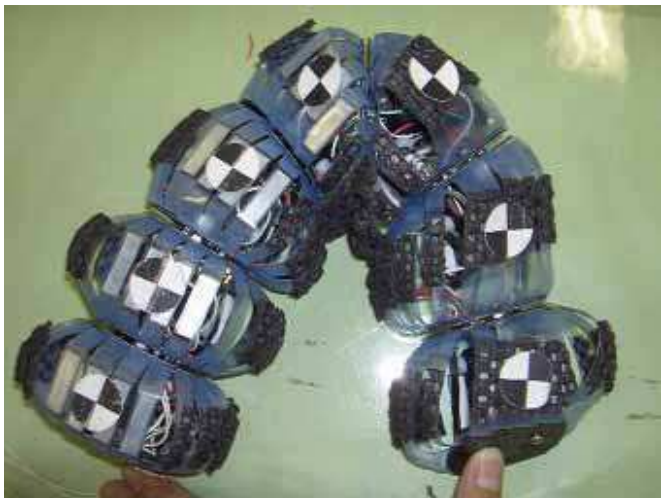

Fig. 8. Units of the robot

\section{Experimental results and discussion}

\subsection{Configuration}

In the experiments, the robot was actuated on a plane surface and in a tube. Vinyl chloride, whose friction coefficient is 0.5 , was used for the experiments. The transparent tube has an inner diameter of $78 \mathrm{~mm}$ and a thickness of $3 \mathrm{~mm}$.

\subsection{Forward movement on the ground and comparison with an actual earthworm}

The forward movement of the robot on the ground was compared with that of an actual earthworm. Fig. 9 shows the locomotion pattern I that contains 7 motions. Four contracted units were present in all motions, and these propagated along the anteroposterior direction between motions. The period of peristaltic crawling was $0.2 \mathrm{~s}$, which is the time between motions. Fig. 10 shows the movement tracks of the robot.

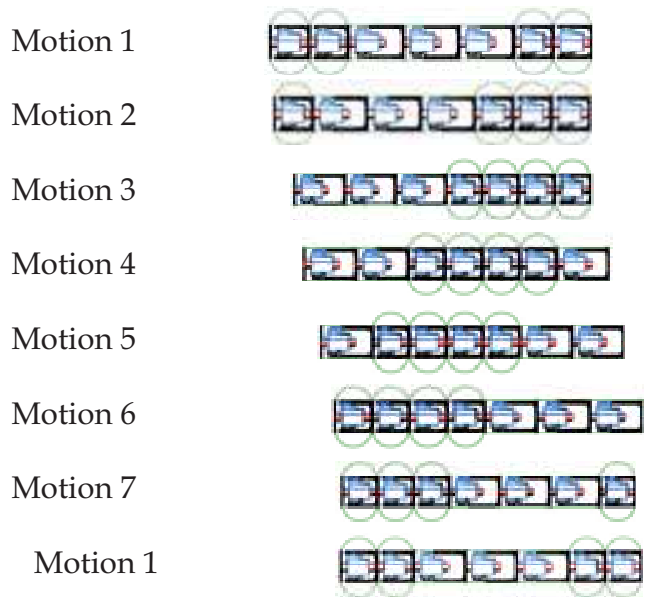

Fig. 9. Pattern I 


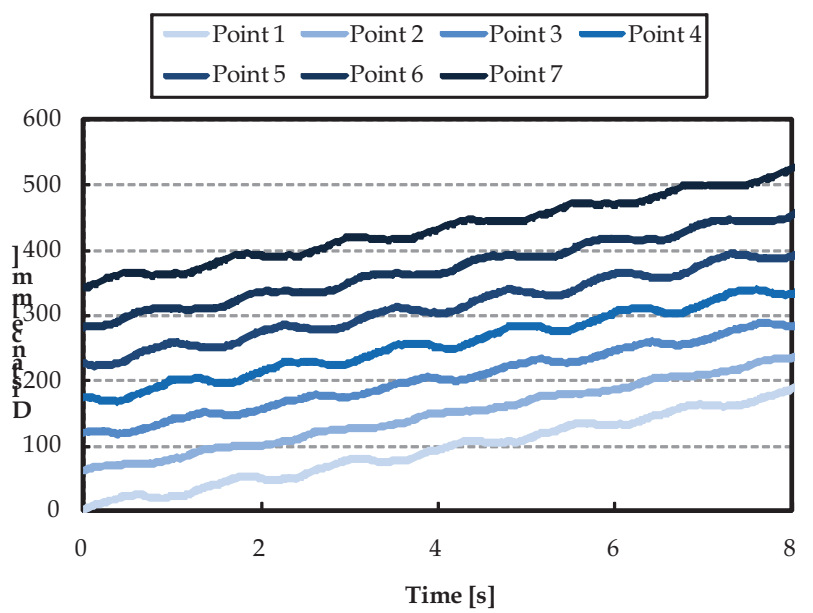

Fig. 10. Movement tracks of flexible robot

Fig. 10 shows that the extension and contraction waves propagate from the anterior to posterior part, resulting in peristaltic crawling of the robot. Compared to Fig. 4, Fig. 10 shows that the robot moved backward since the extension of the anterior part and the contraction of the posterior part occurred simultaneously and that the anterior part was pulled by the posterior part.

\subsection{In the tube}

The angle of the tube was set at $0 \mathrm{deg}, 90 \mathrm{deg}$ and $-90 \mathrm{deg}$. These angles indicate that the robot moves in a horizontal direction, in a vertical upward direction and downward direction, respectively. The locomotion pattern I and a peristaltic crawling period of $0.2 \mathrm{~s}$ were used in the tube experiment.

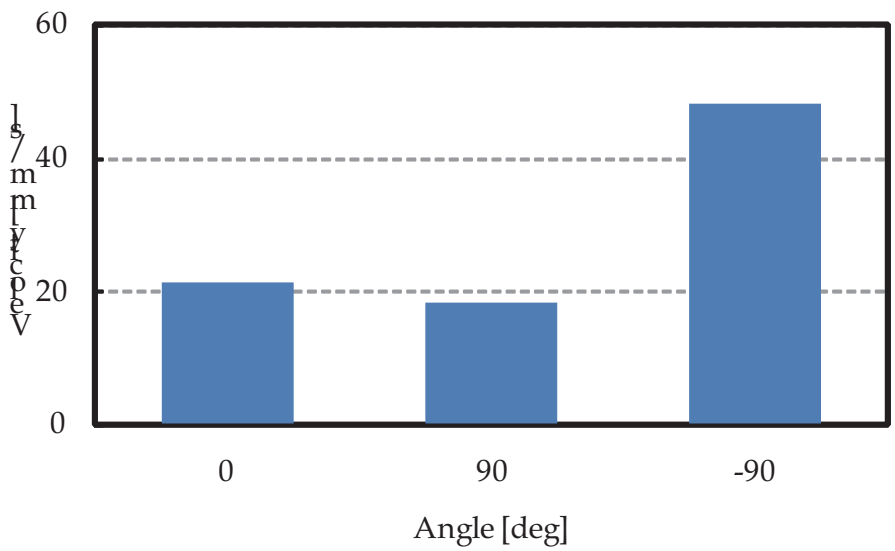

Fig. 11. Velocity in the tube 
Fig. 12 shows the movement tracks of the robot at $0 \mathrm{deg}$, which were analysed to confirm whether the robot moved with peristaltic crawling. The velocity change due to change in the angle of the tube is shown in Fig. 11. Fig. 11 shows that a small difference in the velocity was observed at $90 \mathrm{deg}$, which was affected by the gravity. It was less than the difference we had expected. The velocity at $-90 \mathrm{deg}$ was twice that at $0 \mathrm{deg}$.

\subsection{In the Curved tube}

The flexibility of the robot in a curved tube was examined. Fig. 12 shows the shape of the curved tube, which was made of straight tubes and curved tubes. The robot moves straight, turns 90 deg to the left, moves straight again and then moves up vertically. The locomotion pattern I was used in this experiment.

Fig. 13 shows the photos captured when the robot moved in the curved tube. The robot took $150 \mathrm{~s}$ to move from A to B. We observed that the robot was able to move in the curved tube when the locomotion pattern was used for forward movement and not for turning. Therefore, we confirmed the robot had flexible units.

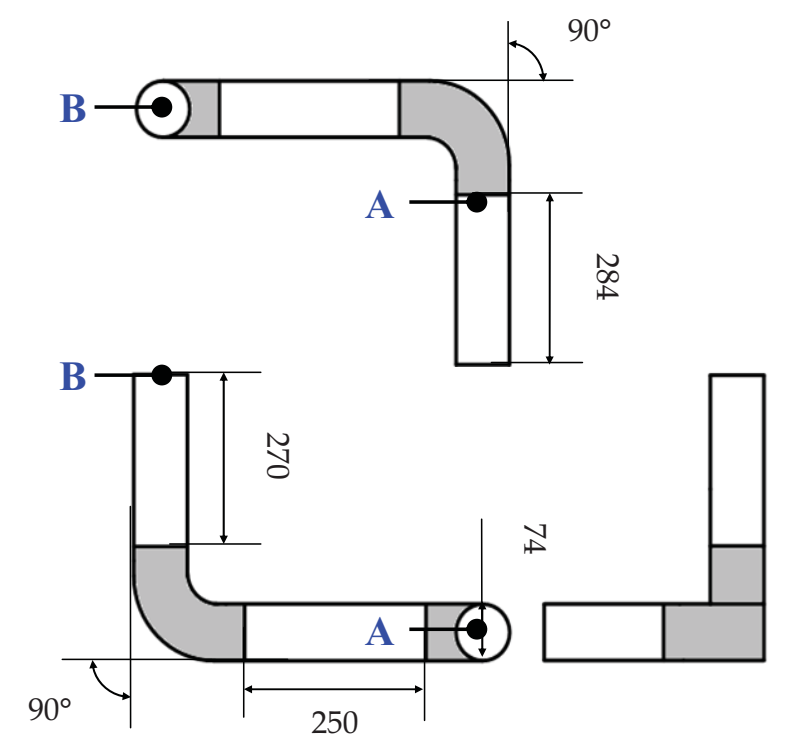

Fig. 12. Shape of the curved tube

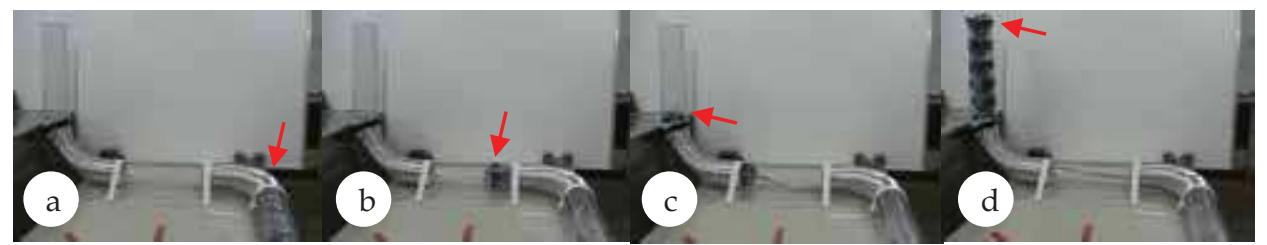

Fig. 13. Movement of the flexible-type robot in the curved tube 


\section{Turning experimental results and discussion}

Turning patterns on the ground were examined. The robot could be turned by having the differences in extension and contraction between right and left segments. The period of peristaltic crawling was $0.2 \mathrm{~s}$. The $\mathrm{Y}$-coordinate, the $\mathrm{X}$ - coor-dinate, and $\theta$ are defined (Fig. 16). The $X$-coordinate is at both the anterior and posterior parts, and $\theta$ the rotation angle from $Y$ to $X$.

\subsection{Turn I and Turn II}

Two patterns were examined to determine which side of the robot needed actuating with peristaltic crawling while turning.

Fig. 14 show turns I and II, respectively. In turn I, the sides of the segments opposite to the turning direction were all extended and those in the same direction were actuated with peristaltic crawling. In turn II, the sides of segments opposite to the turning direction were actuated with peristaltic crawling and those in the same direction were all contracted.

Fig. 15 shows movement tracks of turns I and II. The top track is first unit from the posterior part. Fig. 16 shows transition of the rotational angle.
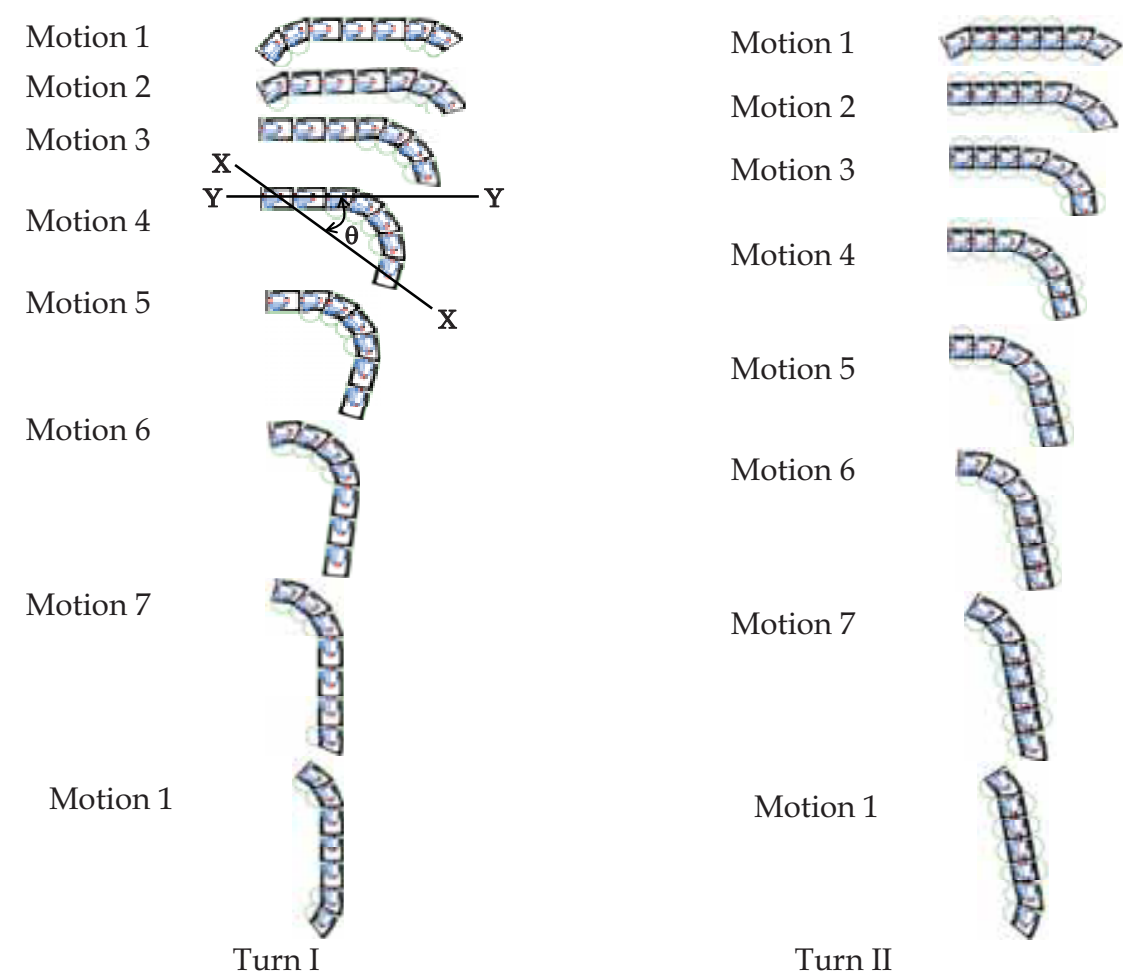

Fig. 14. Turn I and II 


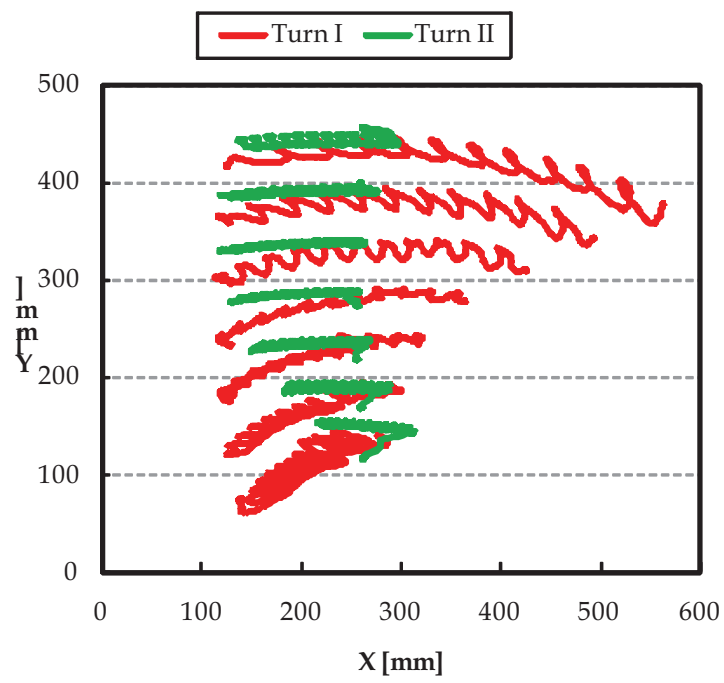

Fig. 15. Movement tracks of Turn I and II

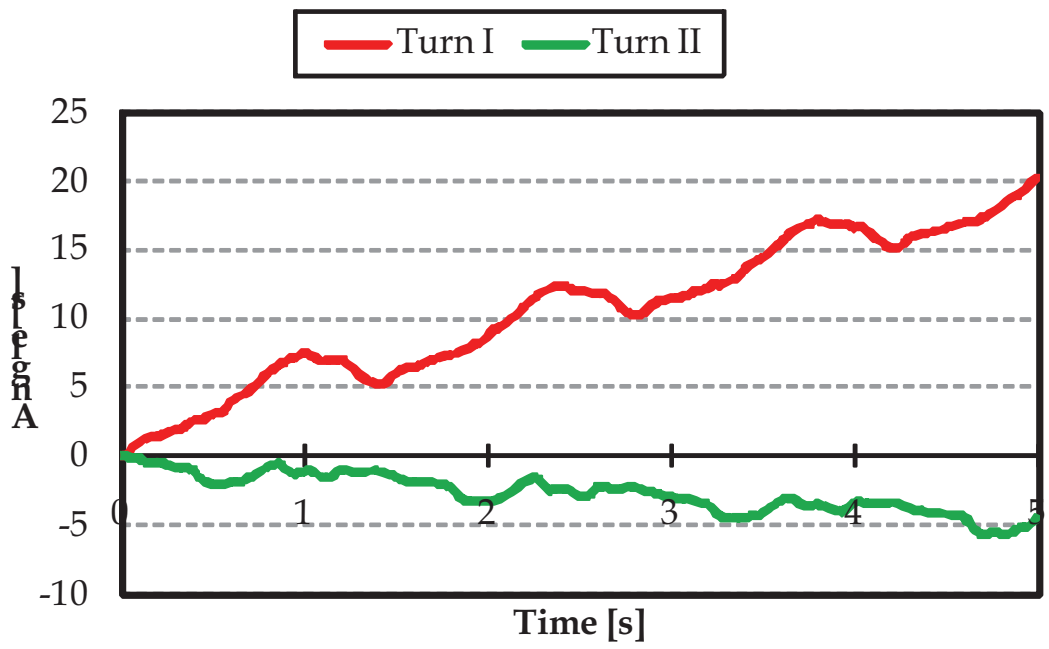

Fig. 16. Transition of the rotational angle

Figs. 15 and 16 show that the robot could turn right using turn I whereas the robot turn left using I. In turn I, the robot moved backward at around 3 deg for every 7 motions, which was because of the short period of peristaltic crawling and increase in the opposite force during expansion of a unit. When the right side of the segments touched the ground in turn II, the robot applied brakes and turned to left by peristaltic crawling. 


\section{Rigid-type robot}

\subsection{Configuration of a Robot with 4 Units}

Fig. 17 shows the configuration of the rigid-type robot. It consists of several units that can extend and contract in the axial direction. The units are connected with a rotational degree of freedom. Hence, it is possible to bend between units. Since peristaltic crawling requires at least three contraction segments, the robot also must consist of at least three units. However, in practice, the robot requires at least four units to change its attitude. The length of 4 units is $279 \mathrm{~mm}$ when fully extended and $231 \mathrm{~mm}$ when fully contracted. The robot weights $283 \mathrm{~g}$.

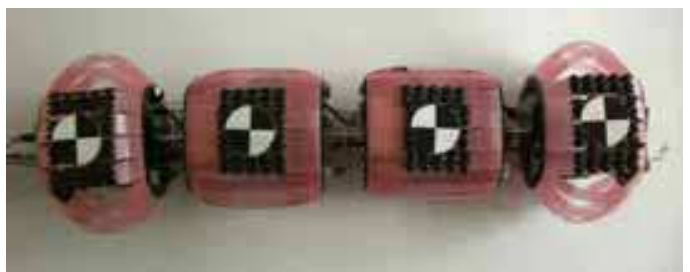

Fig. 17. Rigid-type robot

\subsection{The Structure of a Rigid-type Unit}

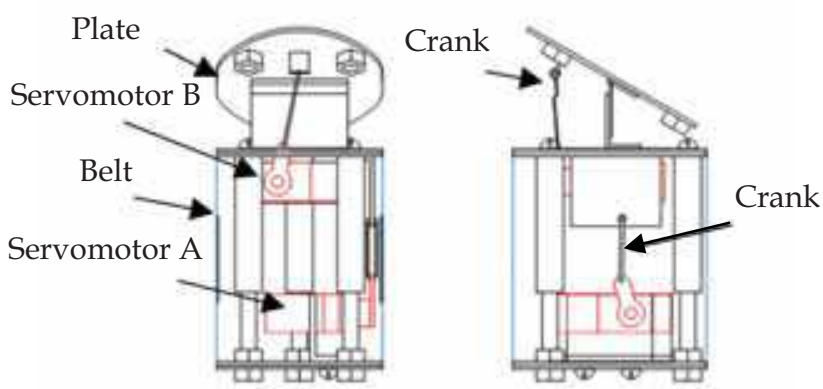

Extension
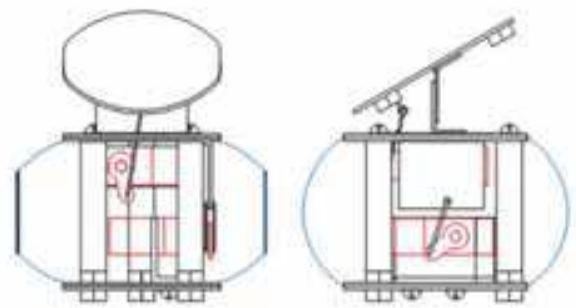

Contraction

Fig. 18. Rigid-type robot 
Fig. 18 shows functions of the rigid-type robot. The units use two servomotors. Servomotor A provides contraction and extension of the unit. The swing motion of the servomotor is converted into a slide motion by a crank mechanism. The unit expands in the radial direction during contraction due to the curvature of belts made from flexible plates. Servomotor $B$ provides bending between the units. A rubber sheet is attached at each belt to increase the friction between the units and the ground when the belts contact the moving surface. Each unit is $65 \mathrm{~mm}$ long and of $40 \mathrm{~mm}$ in diameter, when fully extended, and $50 \mathrm{~mm}$ long and 75 $\mathrm{mm}$ in diameter, when fully contracted. It has a mass of $70 \mathrm{~g}$. The bending angle ranges from -30 to 30 degrees.

\section{Modelling of the robot and simulation}

The developed robot is modeled to evaluate its position and attitude, and a simulation analyses its movement over given routes in 2-dimensional space.

\subsection{Setup coordinates}

The robot and its setup coordinates are shown in Fig. 19. The robot can be divided into a contraction part and a bending part modeled as a slide joint and a rotational joint, respectively. The rotational angle, anterior segment, and posterior segment are paid particular attention - the coordinates of each unit are set from the posterior to the anterior segment, as $\Sigma 1-\Sigma 5$. li is a distance between the origins of $\Sigma \mathrm{i}$ and $\Sigma \mathrm{i}+1$, and $\theta \mathrm{i}$ is the rotational angle at $\Sigma \mathrm{i}+1$. Further, $\Sigma 0$ is the world coordinate system.
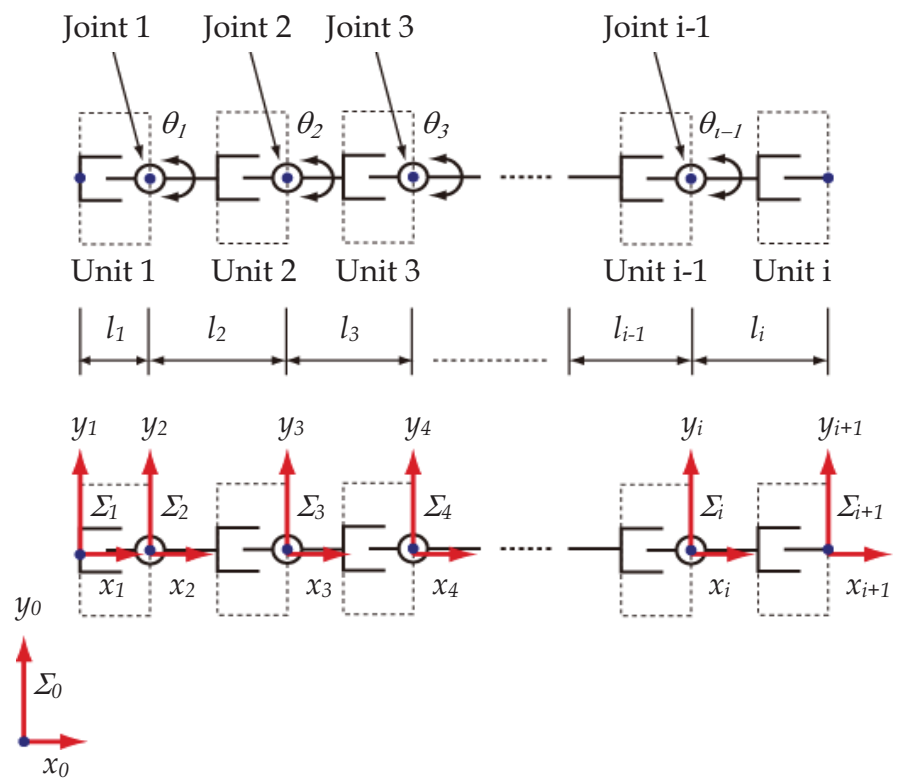

Fig. 19. A set-up of the coordinates of the rigid-type robot 


\subsection{Locomotion patterns for peristaltic crawling}

Fig. 20 shows the pattern of each unit movement required for straight line peristaltic crawling. As shown in this figure, peristaltic clawing is a locomotion pattern whereby contractions of two units propagate along the anteroposterior direction. When the units contract, they come in contact with ground and gain friction. Therefore, the colored contraction unit in each motion (Fig. 20) maintains the position and attitude of the previous motion. This is defined as a basic unit. Hence, when Unit $i$ is the basic unit, $\Sigma i$ should be set as the position and attitude of the previous motion. A contraction unit that has not been colored in Fig. 20 is defined as semi-basic unit. When the attitude of the units is changed to alter the robot's direction, the position and the attitude of the basic and semi-basic units must be fixed. When extension units are between contraction units, such as in Motion 1, the units cannot change their attitude.

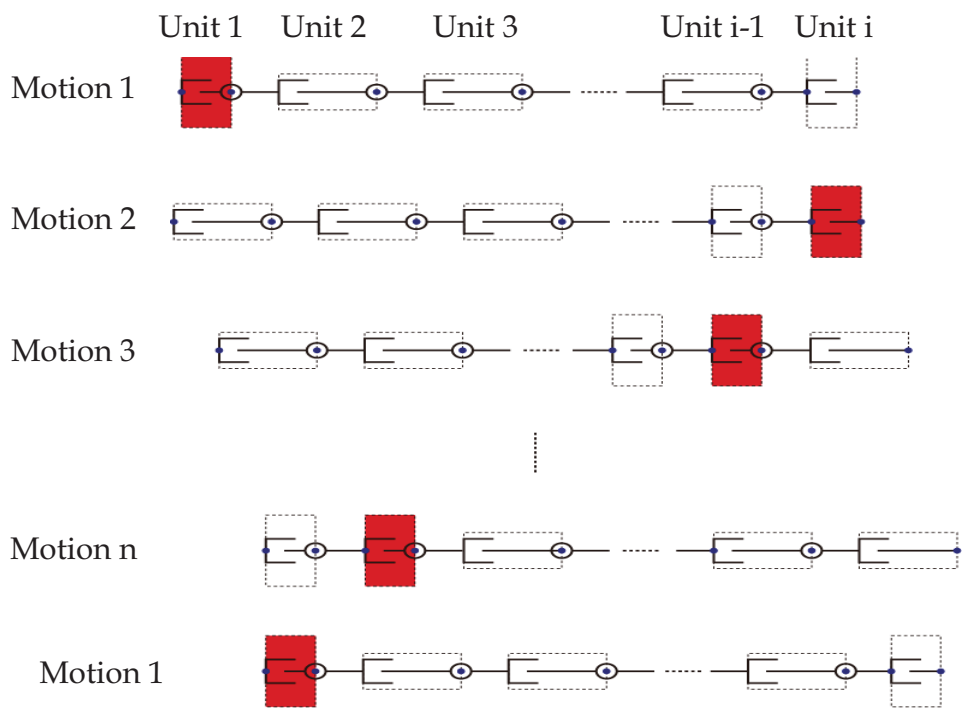

Fig. 20. Locomotion pattern of the rigid-type robot

\subsection{Homogeneous transformation matrix of the robot}

The attitude of the robot observed from the basic unit in each Motion is derived. It is transformed to world coordinate systems to derive a homogeneous transformation matrix of the robot. For instance, a homogeneous transformation matrix for Motion 1 is shown in (1) to (4). The transformation matrix that gives the relationship between the world coordinates $\Sigma 0$ and $\Sigma \mathrm{i}$ is shown in (5).

$$
{ }^{1} T_{2}=\left(\begin{array}{ccc}
c \theta_{1} & -s \theta_{1} & l_{1} \\
s \theta_{1} & c \theta_{1} & 0 \\
0 & 0 & 1
\end{array}\right)
$$




$$
\begin{gathered}
{ }^{2} T_{3}=\left(\begin{array}{ccc}
c \theta_{2} & -s \theta_{2} & l_{2} \\
s \theta_{2} & c \theta_{2} & 0 \\
0 & 0 & 1
\end{array}\right) \\
{ }^{i-1} T_{i}=\left(\begin{array}{ccc}
c \theta_{i-1} & -s \theta_{i-1} & l_{i-1} \\
s \theta_{i-1} & c \theta_{i-1} & 0 \\
0 & 0 & 1
\end{array}\right) \\
{ }^{i} T_{i+1}=\left(\begin{array}{ccc}
1 & 0 & l_{i} \\
0 & 1 & 0 \\
0 & 0 & 1
\end{array}\right) \\
{ }^{0} T_{i}=\left(\begin{array}{ccc}
c \phi & -s \phi & x_{i} \\
s \phi & c \phi & y_{i} \\
0 & 0 & 1
\end{array}\right)
\end{gathered}
$$

\subsection{Setting routes and evaluation functions}

A simulation is performed using 4 units of the robot's derived position and attitude. The contraction pattern of units for robot locomotion is used for the pattern in Fig. 20. The robot can move along the given routes by changing the angle of the joint between units. First, locomotion routes are decided. The given routes that the robot moves along are expressed by the following functions of (6) and (7), representing Route I and Route II. The robot's tail is set at the origin. Route I shows advancement along a straight line, Route II shows a 45degree turn.

$$
\left\{\begin{array}{lr}
y=0 & (x<300) \\
y=x & (x \geq 300)
\end{array}\right.
$$

Next, the evaluation function for motions is discussed. The difference between the robot attitudes and the route in each Motion is examined. Then, the evaluation functions shown in (8) and (9) for Route I and II are set. Each evaluation function shows the distance from the origin of $\Sigma 1-\Sigma 5$ (in world coordinate systems) to the route. The attitude of the robot in each Motion, when these calculation results are the smallest, is defined as the optimal Motion. Here, the range of the angle of each joint is from -30 to 30 degrees (counterclockwise rotation is assumed to be positive), with a resolution of 5 degrees.

$$
E_{0}=\sum_{i=1}^{5}\left|0-y_{i}\right|
$$




$$
\begin{cases}E_{0}=\sum_{i=1}^{5}\left|0-y_{i}\right| & (x<300) \\ E_{1}=\sum_{i=1}^{5}\left|x_{i}-y_{i}\right| & (x \geq 300)\end{cases}
$$

\subsection{Simulation results and discussion}

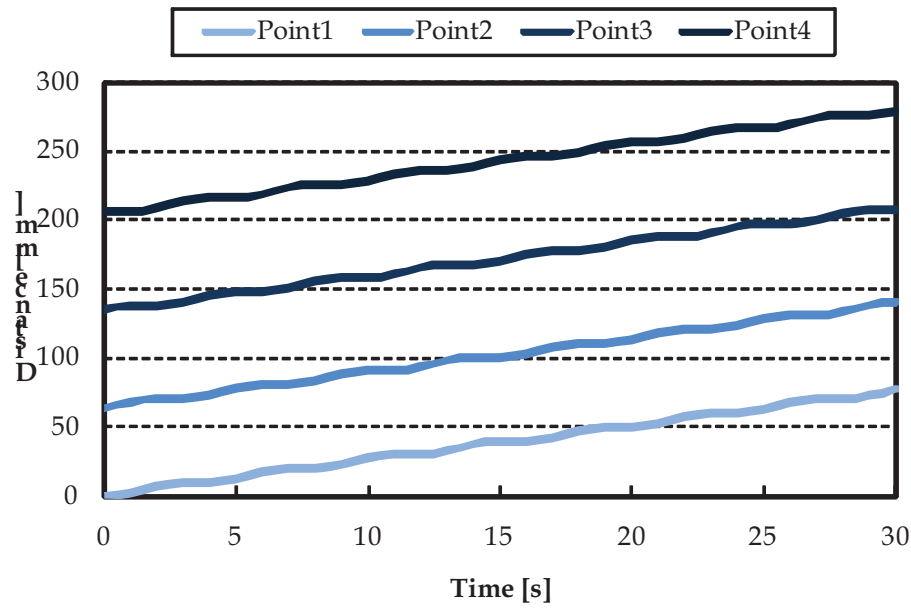

Fig. 21. Simulation results of movement tracks of the robot (Route 1)

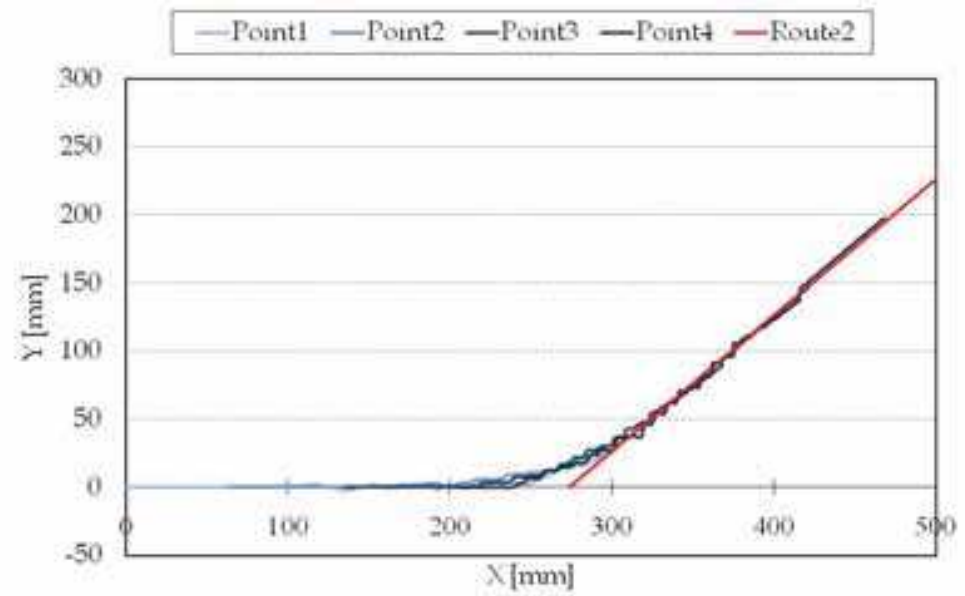

Fig. 22. Simulation results of movement tracks of the robot (Route 2) 
The simulation results for each route are shown in Fig. 21 and Fig. 22. First, consider the simulation results for Route 1. If we look at Fig. 21 (the relationship between the advancement distance and time), it confirms that the robot advances by repeated propagation of contractions from the anterior to posterior part. Thus, the simulation results accurately expressed peristaltic crawling motion. We next discuss the simulation results for Route 2. Fig. 21 shows movement tracks of the robot in 2-dimensional planes. Although this figure shows that the robot's tracks deviate a little from the route, the robot does follow the given route without large deviations. This robot is good at moving in narrow spaces, for straight advancement movement, such as Route 1 . Thus, it is desirable that the robot passes more close to bending and curve paths, such as Route 2. We think that the robot can be made to follow these tracks more faithfully by increasing the joint size.

\section{Experimental results and discussion}

Experiments of the mobile robot using peristaltic crawling motion were performed based on the simulation results. A video of the robot moving on the plane was taken from directly overhead and the tracks of the points marked on each unit were analyzed. The experimental results are shown in Fig. 23-25. From Fig. 23, we can see that the motions of the robot are qualitatively similar to those of the actual earthworm shown in Fig. 4, because the longitudinal wave from the front to the back of is propagated by contracting the units, There is, however, a little slipping caused by the relationship between inertia of the units and its friction force. The tracks are shown for Route 2. Here, the angle of the servomotors is controlled using only the values obtained by simulation results, and no feedback control is applied. Fig. 24 shows that, while the robot's tracks are not quite as close to the path as in the simulation, the robot moves around the route. In particular, the robot starts changing its attitude before reaching the point that the curve function actually changes. The peristalsis crawling robot is moved by using the difference of the friction of the contraction units and the extension units.

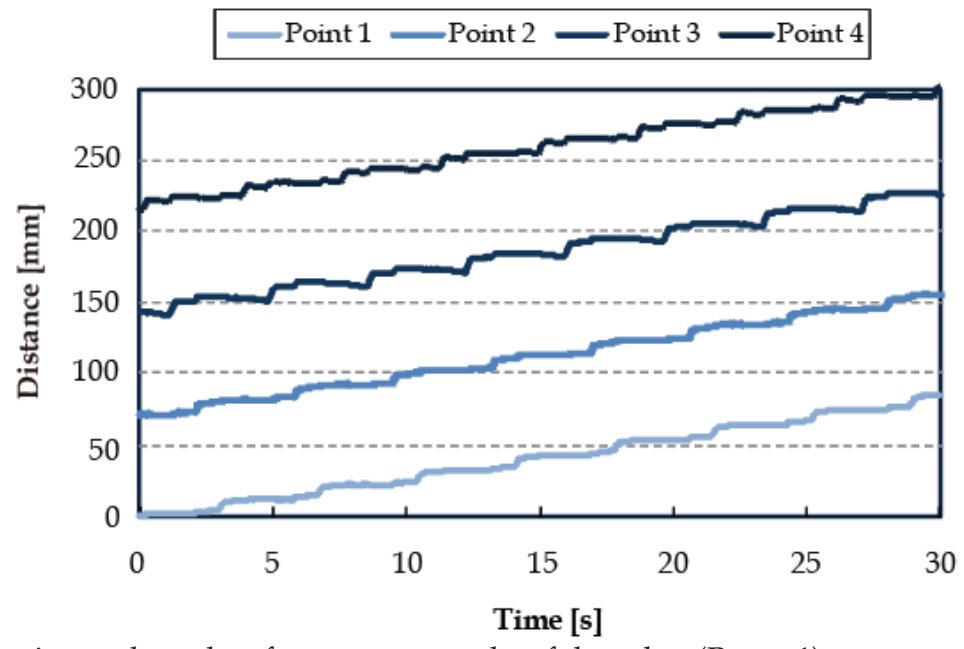

Fig. 23. Experimental results of movement tracks of the robot (Route 1) 


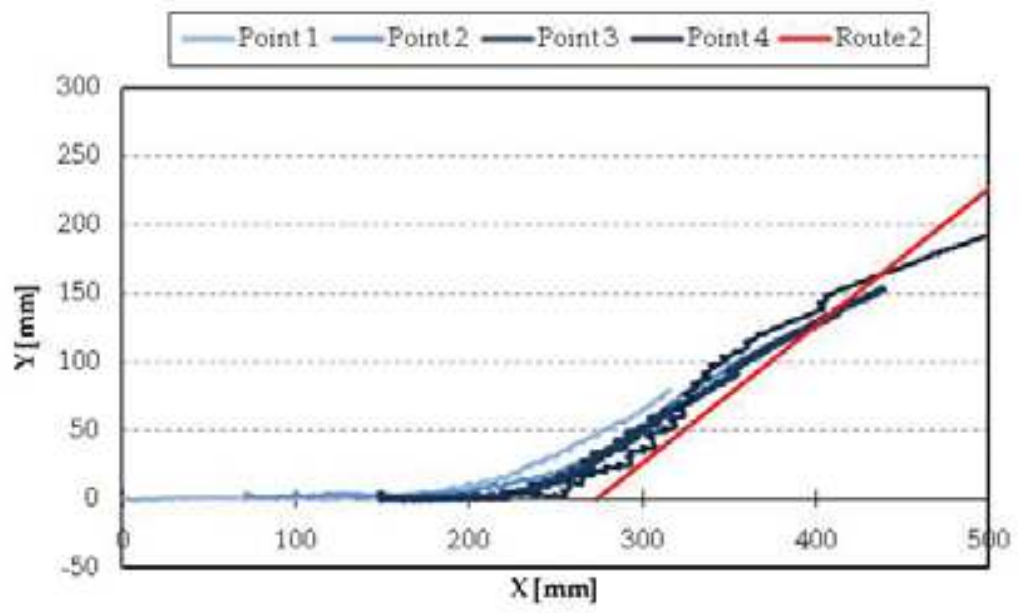

Fig. 24. Experimental results of movement tracks of the robot (Route 2)

Therefore, if the basic and semi-basic units that constitute the contraction units slip, there is a possibility of influencing the distance moved and the bending angle. Also, the robot's weight prevented the belts from achieving full expansion in the radial direction (Fig. 18). Hence, there is a possibility that enough frictional force difference cannot be achieved.

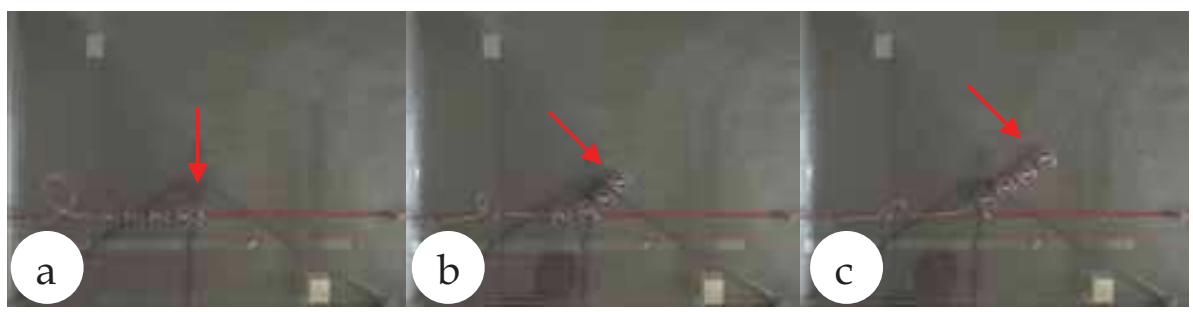

Fig. 25. Experimental results of Route 2

\section{Parallel link robot}

\subsection{Robot}

An underground robot is required to be controlled in 3 dimensions and generative force is large. To meet these requirements, we adopted a parallel link mechanism. Figure 26 shows the structure of a single unit (segment). A parallel link mechanism controls the anterior part in three dimensions and generates a large extraction and contraction force. Figure 27 shows the expansion belts; a sheet is placed between the belts to provide dust protection. Figure 28 shows the robot of 4 units. The length of four units is $300 \mathrm{~mm}$ when fully extended and 220 $\mathrm{mm}$ when fully contracted. The robot weighs $380 \mathrm{~g}$. 


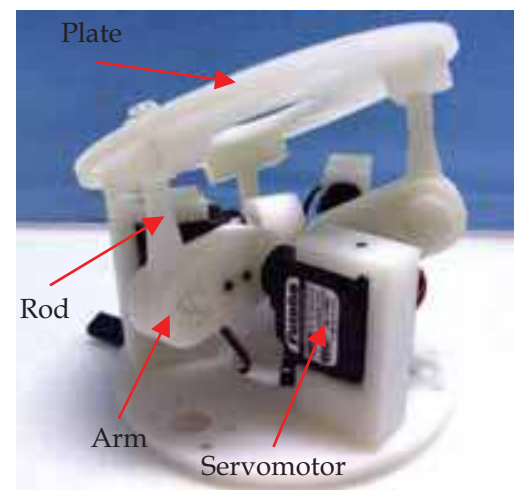

Fig. 26. Internal construction of a single unit

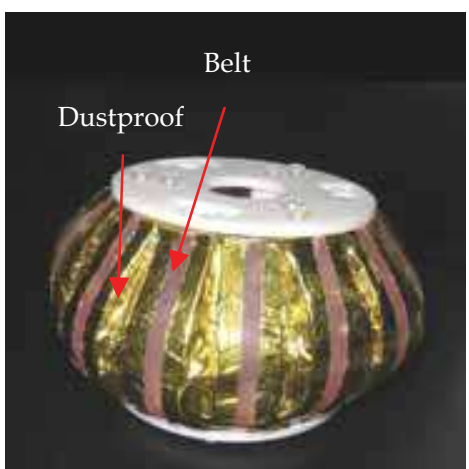

Fig. 27. Expansion belts and dustproof covering

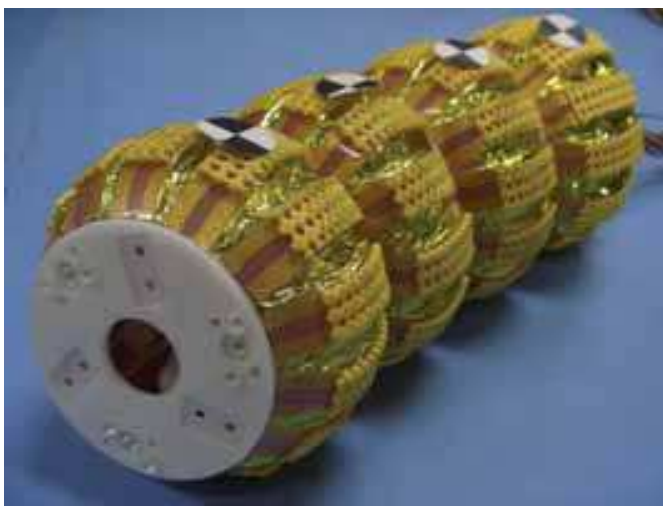

Fig. 28. Prototype of peristaltic crawling robot 


\subsection{Experiments in Dirt}

Fig. 29. Experimental setup
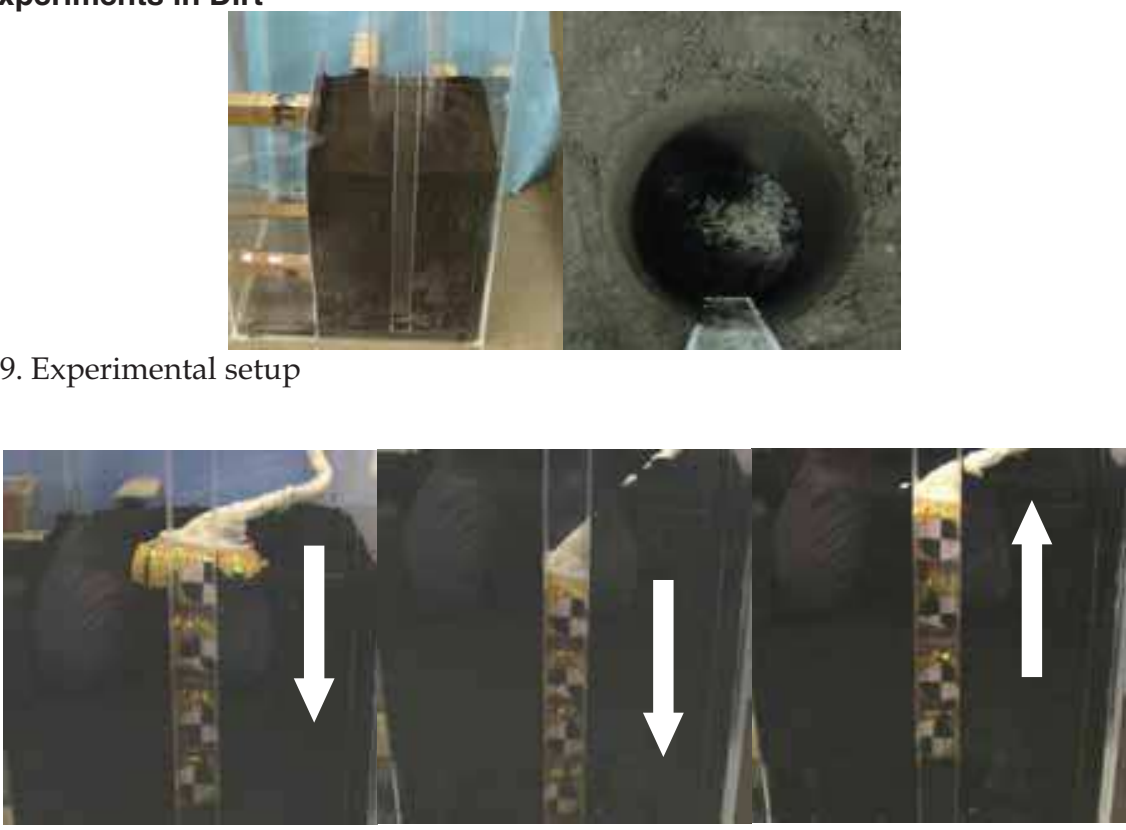

Fig. 30. Moving in dirt

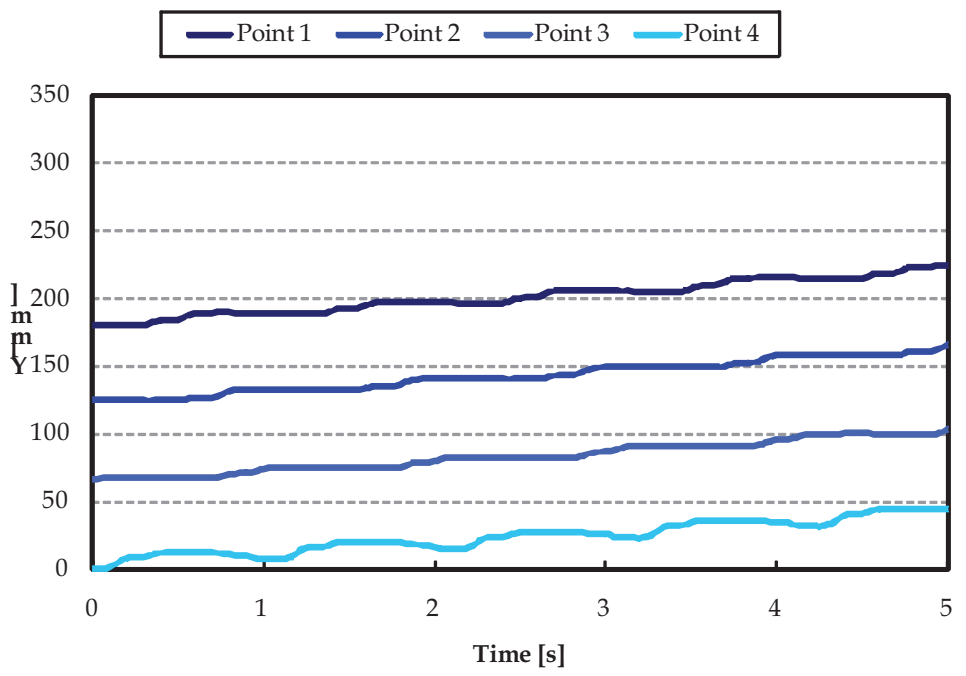

Fig. 31. Locomotion track of the robot in $\operatorname{dirt}\left(90^{\circ}\right)$

The robot was equipped with a dustproof covering so that we could conduct the experiments in dirt. Figure 29 shows the experimental setup. As, the prototype robot did not have 
an excavation unit, we first set the tube used for the climbing experiments. We then placed black dirt around the tube, and finally withdrew the tube. Through experiments in dirt, we confirmed that the robot can move vertically both upwards and downwards in perforated dirt (Fig.30). Figure 31 depicts the movement tracks of the robot at $90^{\circ}$ in dirt. We confirmed that the robot was able to move in the dirt.

\section{Conclusion}

In this study, we developed two types of peristaltic crawling robots based on an actual earthworm, and introduced one of the applications.

1, Flexible-type of robot

2, Rigid-type of robot

Talking of the flexible-type robot; the movement of the flexible type-robot on the ground and in a narrow pipe was compared to that of an actual earthworm, and it was confirmed that the movement of the robot resembled that of an actual earthworm. In addition, the robot could move in a curved tube. Thus, we successfully developed a peristaltic crawling earthworm robot with flexibility. In turn I, the robot could turn, whereas in turn II, the robot could not turn to the right direction.

Talking of the rigid-type of robot; We proposed a locomotion strategy in 2-dimensional space, based on peristaltic crawling. The robot was modeled to determine its positions and attitude. We performed a simulation of its movements on given routes. The simulation results showed good performance. Experiments with the mobile robot, using peristaltic crawling, were carried out, based on the simulation results. Although the robot did not track the path as closely as the simulation, the robot moved around the routes.

An application introduced was constituted of several parallel links for meeting the requirements of an underground robot. We had experiments in perforated dirt and the experiments were successful.

In the future, these robots will be improved and several sensors will be attached for obtaining surrounding data. In addition, it is necessary that the robots have excavation mechanisms for an underground explorer.

\section{References}

Mangan, E.V.; Kingsley, D.A.; Quinn, R.D. \& Chiel, H.J. (2002). Development of a peristaltic endoscope, Proceedings of IEEE International Conference on Robotics and Automation, pp. 3282-3287, Washington DC, May 2002

Menciassi, A.; Gorini, S.; Pernorio, G. \& Dario, P. (2004). A SMA actuated artificial earthworm, Proceedings of IEEE International Conference on Robotics and Automation, pp. 347-352, LA, April 2004, New Orleans

Zuo, J.; Yan, G. \& Gao, Z. (2005). A micro creeping robot for colonoscopy based on the earthworm, Journal of Medical Engineering ETechnology, Vol. 29, 1, pp. 1-7

Kim B,; Lee MG,; Lee YP, et al. (2006). An earthworm-like micro robot using shape memory alloy actuator, Sensors and actuators. A, Physical, Vol. 125 No. 2: pp. 429-437, 09244247

Norihiko, S. \& Taro N. (2004). A Prototype of Peristaltic Robot Using Pneumatic Artificial Muscle, Intelligent autonomous system, No. 8, pp. 85-95 
Norihiko, S. \& Taro, N. (2004). Development of peristaltic-crawling robot using magnetic fluid on the basis of locomotion mechanism of earthworm, Smart material and structure, Vol. 13, No. 3, pp. 566-569

Taro, N.; Takashi, K.; Tomohide, I. \& Yoichi, M. (2006). Development of a Peristaltic Crawling Robot Using Servo Motors Based on the Locomotion Mechanism of Earthworms, Proceedings of IEEE International Conference on Robotics and Automation, pp. 4342-4344

Taro, N.; Takashi, K.; Tomohide, I. \& Yoichi, M. (2006). Development of a Peristaltic Crawling Robot Based on Earthworm Locomotion, Journal of Robotics and Mechatronics, Vol.18, No. 3, pp .299-304

Alexander, R. M. (1992). Exploring Biomechanics, Animals in Motion, W.H. Freeman and Company, Scientific American Library, 071675035X, New York

Sugi. H. (1977). Evolution of Muscle Motion, the University of Tokyo Press, pp. 72, 1977 (in Japanese

Taro, N. \& Tomihide, I. (2008). Locomotion Strategy for a Peristaltic Crawling Robot in a 2Dimensional Plane, Proceedings of IEEE International Conference on Robotics and Automation, pp. 238-243, USA, May 2008, Pasadena

Hayato, O,; Takeshi, H. \& Taro, N. (2008). Locomotion and Turning Patterns of a Peristaltic Crawling Earthworm Robot Composed of Flexible Units, Proceedings of IEEE International Conference on Robotics and Automation, pp. 1630-1635, France, September 2008, Nice 


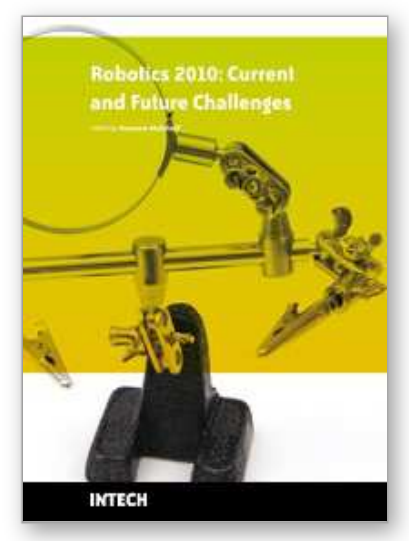

\section{Robotics 2010 Current and Future Challenges}

Edited by Houssem Abdellatif

ISBN 978-953-7619-78-7

Hard cover, 494 pages

Publisher InTech

Published online 01, February, 2010

Published in print edition February, 2010

Without a doubt, robotics has made an incredible progress over the last decades. The vision of developing, designing and creating technical systems that help humans to achieve hard and complex tasks, has intelligently led to an incredible variety of solutions. There are barely technical fields that could exhibit more interdisciplinary interconnections like robotics. This fact is generated by highly complex challenges imposed by robotic systems, especially the requirement on intelligent and autonomous operation. This book tries to give an insight into the evolutionary process that takes place in robotics. It provides articles covering a wide range of this exciting area. The progress of technical challenges and concepts may illuminate the relationship between developments that seem to be completely different at first sight. The robotics remains an exciting scientific and engineering field. The community looks optimistically ahead and also looks forward for the future challenges and new development.

\section{How to reference}

In order to correctly reference this scholarly work, feel free to copy and paste the following:

Hayato Omori, Taro Nakamura, Tomohide Iwanaga and Takeshi Hayakawa (2010). Development of Mobile Robots Based on Peristaltic Crawling of an Earthworm, Robotics 2010 Current and Future Challenges, Houssem Abdellatif (Ed.), ISBN: 978-953-7619-78-7, InTech, Available from:

http://www.intechopen.com/books/robotics-2010-current-and-future-challenges/development-of-mobile-robotsbased-on-peristaltic-crawling-of-an-earthworm

\section{INTECH}

open science | open minds

\author{
InTech Europe \\ University Campus STeP Ri \\ Slavka Krautzeka 83/A \\ 51000 Rijeka, Croatia \\ Phone: +385 (51) 770447 \\ Fax: +385 (51) 686166 \\ www.intechopen.com
}

\section{InTech China}

Unit 405, Office Block, Hotel Equatorial Shanghai

No.65, Yan An Road (West), Shanghai, 200040, China

中国上海市延安西路65号上海国际贵都大饭店办公楼 405 单元

Phone: +86-21-62489820

Fax: +86-21-62489821 
(C) 2010 The Author(s). Licensee IntechOpen. This chapter is distributed under the terms of the Creative Commons Attribution-NonCommercial-ShareAlike-3.0 License, which permits use, distribution and reproduction for non-commercial purposes, provided the original is properly cited and derivative works building on this content are distributed under the same license. 